\title{
Correction to: Advancing Pharmacotherapy Development from Preclinical Animal Studies
}

\author{
Mark Egli
}

\section{Correction to: \\ (C) Springer International Publishing AG 2018 Handbook of Experimental Pharmacology, https://doi.org/10.1007/164_2017_85}

In the 7th line from the bottom of the Abstract the word nociception appears incorrect. It should read nocioceptin. In section 3.4 on the third line of 2 nd paragraph the word nociception appears incorrect. It should read nocioceptin. The original chapter was corrected. 